\title{
Investigating the Three-Dimensional Microstructural Characteristics of Lithium- Sulfur Electrodes with X-Ray Micro-Tomography
}

\author{
C. Tan $^{\mathrm{a}}$, S. R. Daemi ${ }^{\mathrm{a}}$, D. J. L. Brett, ${ }^{\mathrm{a}}$ and P. R. Shearing ${ }^{\mathrm{a}}$ \\ ${ }^{a}$ Electrochemical Innovation Lab, Department of Chemical Engineering, University \\ College London, London WC1E 7JE, United Kingdom
}

The Li-S battery has the potential to replace Li-ion batteries in high capacity and high gravimetric energy density applications, but certain technical challenges result in diminished cycle life and have hindered its commercialization. Lab-scale X-ray microtomography was performed on an elemental sulfur electrode and a miniaturized in-situ tomography Li-S cell, and the 3D visualization achieved with this technique provides a wealth of information, improving understanding of the evolution of morphological and microstructural parameters of the different phases. As X-ray imaging is inherently non-destructive, it enables these parameters to be observed in-situ as a function of cycle life and state of charge. The inherently anisotropic nature of active material dissolution, recrystallization and other degradation processes within the electrode necessitates a three-dimensional approach to enable their visualization and quantification, and X-ray tomography is a useful tool in informing the development of more optimal electrode designs.

\section{Introduction}

The Li-S battery offers a theoretical capacity of $1672 \mathrm{mAh} \mathrm{g}^{-1}$ (1), almost tenfold greater than the gravimetric capacity of current Li-ion battery technology (155 $\left.\mathrm{mAh} \mathrm{g}^{-1}\right)$ (2), illustrating its potential in replacing Li-ion batteries in high capacity and high gravimetric energy density applications. The elemental sulfur used in Li-S batteries is naturally abundant and relatively non-toxic compared to the transition metal oxides used in conventional Li-ion batteries. However, the technical challenges due to the complex electrochemistry behind Li-S technology, poor electrical properties of sulfur and complex degradation mechanisms occurring within the Li-S cell have hindered its commercialization. Through the 3D visualization of the sulphur electrode and Li-S cell, a wealth of information about the morphology and microstructure of the electrodes as a function of state of charge (SoC) and cycle life can be obtained. In addition to extracting microstructural parameters such as porosity and tortuosity (3-7), failure mode analysis can be performed throughout electrode depth, and macroscopic electrochemical behaviour can be correlated with microstructure.

The application of in-situ X-ray micro-tomography (micro-CT) as a technique to characterize Li-S batteries as a function of cycle life has been demonstrated earlier by our group (8), and various improvements to the elemental sulfur electrode and tomography cell design have been made in order to improve the image quality of the acquired volume, reduce acquisition time and better approximate a typical thin film sulfur electrode in 
terms of mass loading and electrode thickness. In this work, elemental sulfur with a smaller particle size was used to make a sulfur electrode, along with a lower mass loading of sulfur compared to the electrode used in the earlier publication (8). One of the aims of reducing sulfur particle size and mass loading within the cathode was to be better able to track individual sulfur particles across cycle number and state of charge. The improvement of signal to noise ratio will ensure that advanced image analysis techniques such as digital volume correlation (DVC) can be more easily and more reliably applied to the tomography results. These advanced image analysis techniques will be explored in future work, and the preliminary results presented in this work are a proof of concept on the improvements that a more relevant sulfur electrode and optimized tomography cell design will bring.

Ex-situ micro-CT of an elemental sulfur electrode was performed to characterize the microstructure of the electrode, and this electrode was cut to size and characterized insitu in an improved tomography cell made of Polyether ether ketone (PEEK). Interrupted in-situ micro-CT of the Li-S PEEK tomography cell was then performed within the same spatial volume at the uncycled, fully discharged and recharged state. This has revealed, for the first time, the complete dissolution of sulfur particles and recrystallization at different spatial positions within the electrode thickness during the first cycle.

\section{Methodology}

\section{Elemental sulfur cathode}

Elemental sulfur (325 mesh, Alfa Aesar), conductive carbon (Super P Li, Alfa Aesar) and poly-vinylidene fluoride (PVDF Solef 5130, Solvay) were dispersed (60:20:20wt\%) in 1-methyl-2-pyrrolidone (NMP, Sigma Aldrich), stored over $3 \AA$ molecular sieves to remove residual moisture, in a high shear tube drive mixer (IKA Turrax, IKA Corporation). The resulting slurry was then cast on aluminum foil (MTI Corporation) by a film applicator (4 sided fixed gap Bird-type film applicator, TQC B.V.) with gap clearances of either $150 \mu \mathrm{m}$ or $200 \mu \mathrm{m}$.

\section{Electrolyte}

1,3-dioxolane (DOL, Sigma Aldrich) and tetraethylene glycol dimethylether (TEGDME, Sigma Aldrich), stored over $3 \AA$ molecular sieves to remove residual moisture, were stirred in a 1:2 ratio by volume of DOL:TEDGME with anhydrous lithium bis(trifluoromethanesulfonyl)imide salt (LiTFSI, Sigma Aldrich) to form a 1M solution of LiTFSI in DOL/TEGDME. Coulometric Karl Fisher titration (C20 Coulometric KF Titrator, Mettler Toledo) of the electrolyte mixture did not detect any traces of moisture. The electrolyte was stored in a dark air tight bottle in an argon filled glovebox (LABStar, MBraun) with $\mathrm{H} 2 \mathrm{O}$ and $\mathrm{O} 2$ levels kept below $0.5 \mathrm{ppm}$.

\section{Electrochemical characterization}

Electrochemical characterization of the elemental sulfur electrode was performed on the sulfur cathode in a 1/2" Swagelok half-cell configuration with lithium foil punched to $10 \mathrm{~mm}$ as the anode, and glass fibre (Whatman GF/D) punched to $13 \mathrm{~mm}$ as the separator. The elemental sulfur electrode as described earlier was punched into $10 \mathrm{~mm}$ discs, and 
the electrolyte used was $1 \mathrm{M}$ LiTFSI in TEGDME/DOL as described earlier. The electrochemical cell was cycled between $1.5-2.8 \mathrm{~V}$ in a galvanostat / potentiostat (Interface 1000, Gamry Instruments) at a C/10 rate based on the theoretical specific capacity of sulfur, equivalent to a current rate of $0.25 \mathrm{~mA} \mathrm{~cm}$ or $167 \mathrm{~mA} \mathrm{~g}^{-1} \mathrm{~S}$ for 20 cycles. The same charge/discharge conditions were applied to the tomography cell, and the cell was imaged when uncycled, after the $1^{\text {st }}$ discharge and again after the $1^{\text {st }}$ charge.

\section{$\underline{\text { In-situ tomography cell }}$}

The in-situ 'tomography cell' was assembled in 1/32' PEEK unions (VIZU.5TFPK, VICI AG) with lithium foil punched to $0.8 \mathrm{~mm}$ as the anode, and glass fibre (Whatman GF/D) punched to $1 \mathrm{~mm}$ as the separator. The elemental sulfur electrode as described earlier was punched into $0.75 \mathrm{~mm}$ discs with a biopsy punch, and the electrolyte used was $1 \mathrm{M}$ LiTFSI in TEGDME/DOL as described earlier.

\section{$\underline{\text { X-ray micro-tomography, reconstruction and quantification }}$}

$\mathrm{X}$-ray micro-tomography was performed on the elemental sulfur cathode sample and the in-situ tomography cell using the ZEISS Xradia 520 Versa. For the ex-situ scan, the pixel size was ca. $186 \mathrm{~nm}$ and field of view was ca. $340 \mu \mathrm{m}$, whilst for the in-situ scans, the pixel size was ca. $382 \mathrm{~nm}$ and the field-of-view was ca. $390 \mu \mathrm{m}$. For each set of tomographic data, a tube voltage of $80 \mathrm{kV}$ was used, and 1601 radiographic projections were obtained at discrete angular steps. The radiographic projections collected were reconstructed using a cone-beam filtered back projection algorithm (XMReconstructor, Carl Zeiss Inc.) to produce a set of tomographic slices making up a cylindrical volume.

3D volume renderings of all tomography datasets were generated in Avizo (FEI Company). For image comparison at different states of charge, the in-situ datasets were rotated such that visual markers identified within each dataset are oriented in the same spatial positions. $2 \mathrm{D}$ reconstructed virtual slices in the plane parallel to the current collector were then extracted from each dataset for visual comparison. 


\section{Results}

Electrochemical performance of elemental sulfur electrode

The elemental sulfur electrode exhibited an initial discharge capacity of $0.97 \mathrm{mAh}$ $\mathrm{cm}^{-2}$ or ca. $694 \mathrm{mAh} \mathrm{g}^{-1} \mathrm{~S}$ when discharged at a C/10 rate, with 2 discharge plateaus at ca. $2.4 \mathrm{~V}$ and $2.0 \mathrm{~V}$. This is about $42 \%$ of the theoretical gravimetric specific capacity of sulfur at $1672 \mathrm{mAh} \mathrm{g}^{-1} \mathrm{~S}$. As calculated by Xu et al. (9), the second discharge plateau, corresponding to the conversion of intermediate polysulfide species to insoluble $\mathrm{Li}_{2} \mathrm{~S}$, should account for about $75 \%$ of the theoretical discharge capacity of sulfur. However, as shown in Figure 1(a), the second discharge plateau at $2.0 \mathrm{~V}$ is significantly shorter, hence contributing to the lower overall discharge capacity seen in this cell. This is due to the lack of polysulfide containment within the sulfur cathode, where soluble polysulfides can freely diffuse into the bulk electrolyte. The tomography dataset at the discharged state will reveal further insights into the poor discharge capacity of this elemental sulfur electrode formulation.
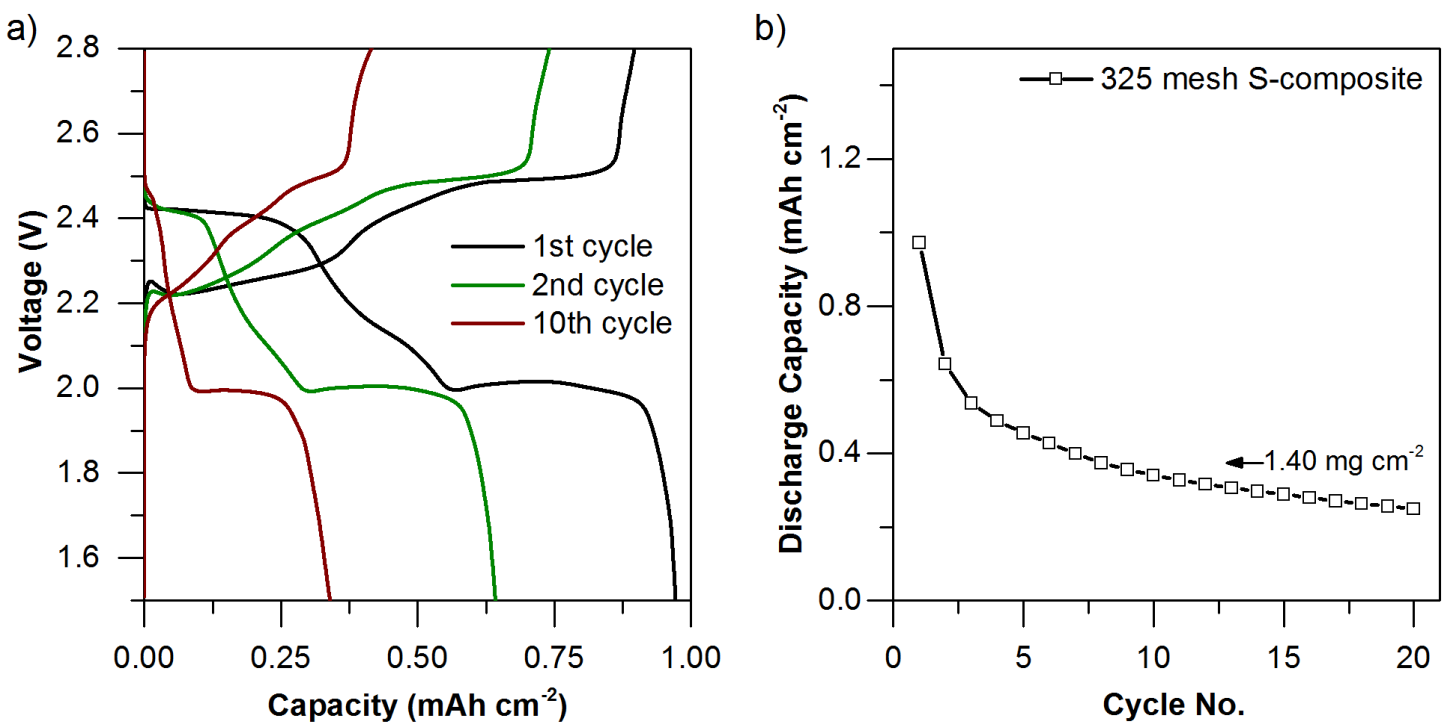

Figure 1. (a) Charge/discharge profile of electrochemical cell in terms of areal capacity over the $1^{\text {st }}, 2^{\text {nd }}$ and $10^{\text {th }}$ cycle. (b) Discharge capacity of electrochemical cell over 20 cycles. Current rate used is equivalent to C/10 based on theoretical specific capacity of sulfur, i.e. ca. $167 \mathrm{~mA} \mathrm{~g}^{-1} \mathrm{~S}$ or $0.25 \mathrm{~mA} \mathrm{~cm}^{-2}$.

Figure 1(b) shows the discharge capacity of the sulfur cathode over 20 cycles, which decreased from $0.97 \mathrm{mAh} \mathrm{cm}^{-2}$ in the $1^{\text {st }}$ cycle to $0.25 \mathrm{mAh} \mathrm{cm}^{-2}$. The poor capacity and rapid cell degradation are to be expected as common mitigation strategies such as sulfur trapping, lithium anode protection and additives such as $\mathrm{LiNO}_{3}$, were not used in this work. Instead, this work seeks to provide a fundamental understanding into the mechanisms behind sulfur dissolution and recrystallization as a function of state of charge, and to improve this technique for the study of more technologically relevant electrode and cell designs. 


\section{$\underline{\text { Volume rendering of elemental sulfur electrode }}$}

As shown in Figure 2, the sulfur, carbon binder and pore phases are clearly distinguishable based on their absorption contrast values, where sulfur is more attenuating and hence appears brighter compared to the carbon binder phase. The volume rendering of the elemental sulfur electrode acquired ex-situ, reveals a generally well distributed sulfur and carbon binder coating along the aluminum current collector. Although not within the scope of this article, morphological parameters such as sulfur phase fraction can be directly extracted from image data, along with porosity and tortuosity information.

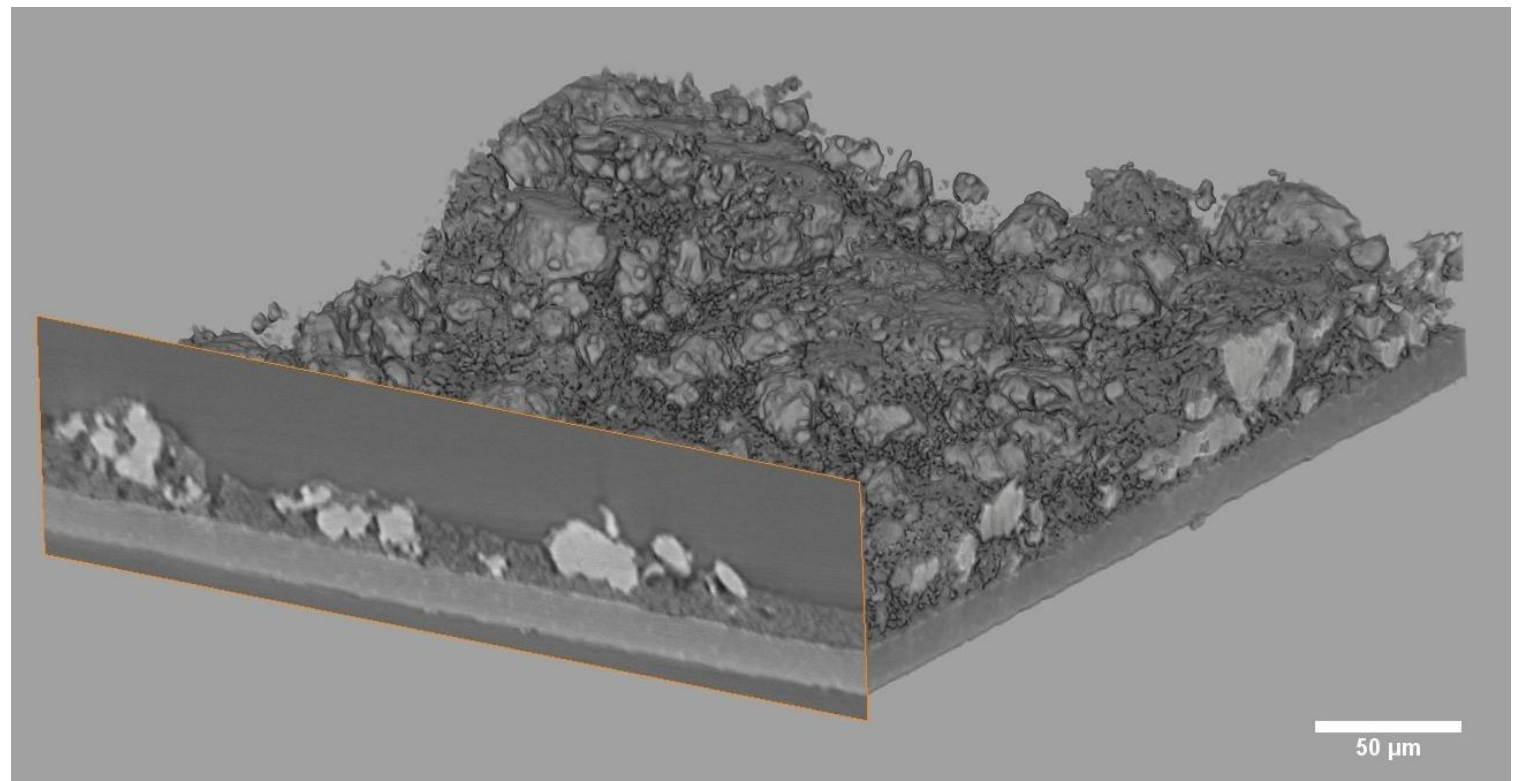

Figure 2. Volume rendering of elemental sulfur electrode with a 2D reconstructed virtual slice showing a cross-section of the electrode.

\section{PEEK tomography cell}

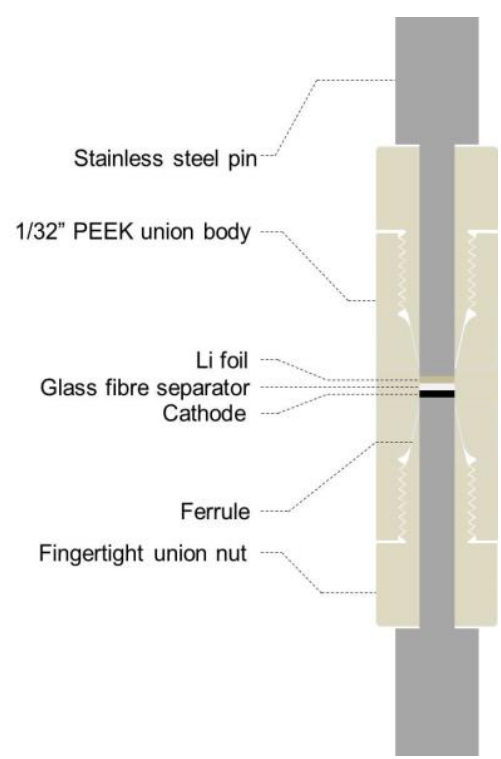

Figure 3. (a) Schematic of the PEEK tomography cell, showing various components within the cell including the location of electrodes. 
The PEEK tomography cell, illustrated in Figure 3, has been demonstrated to double the pixel resolution achievable and halve the total acquisition time in the ZEISS Xradia 520 Versa compared to the previous 1/8" PFA Swagelok cell design used earlier (8). As shown in Figure 4, significant improvement in the signal to noise ratio is observable, showing finer grain detail within individual sulfur particles.

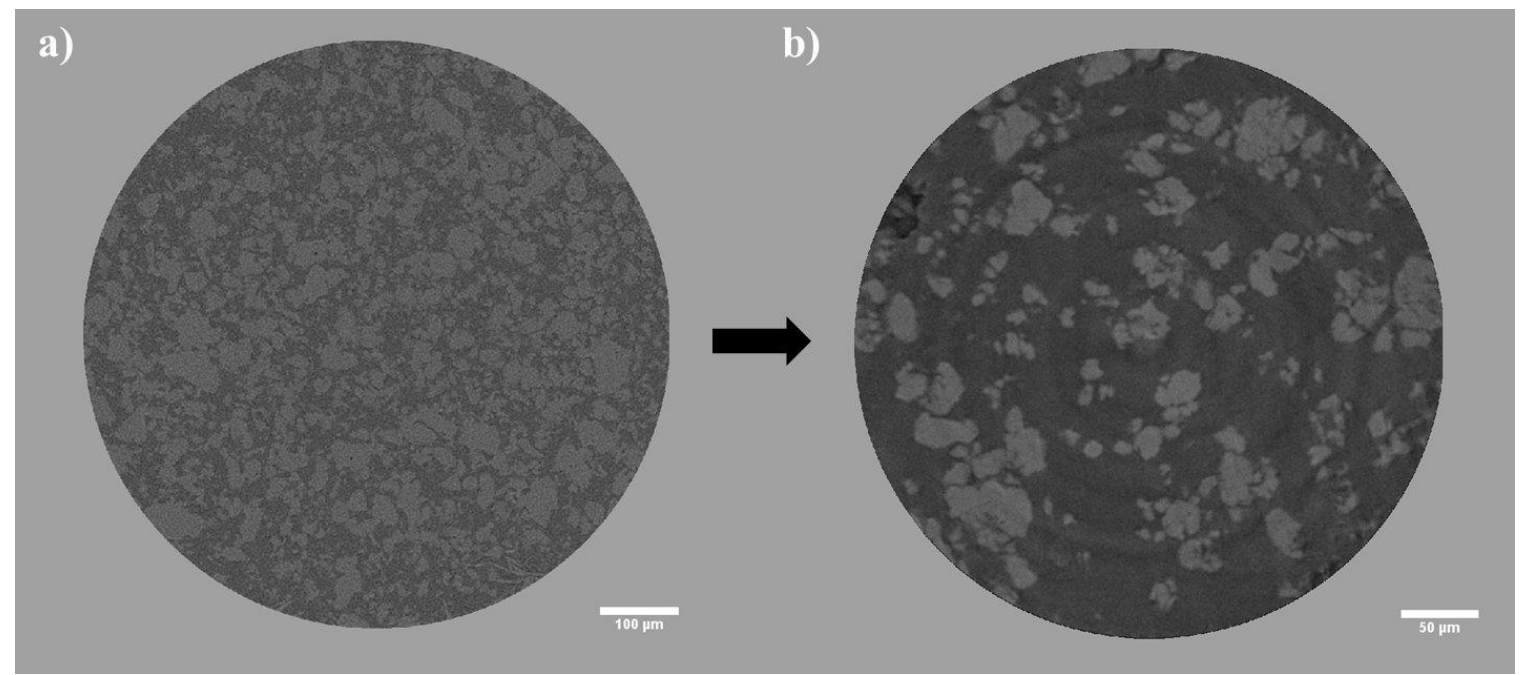

Figure 4. (a) 2D reconstructed virtual slice of higher mass loading elemental sulfur electrode in previous $1 / 8$ " Swagelok tomography cell design. (b) 2D reconstructed virtual slice of elemental sulfur electrode (as described in this work) in current 1/32" PEEK tomography cell design

Electrochemical performance of elemental sulfur electrode in tomography cell

\section{a)}

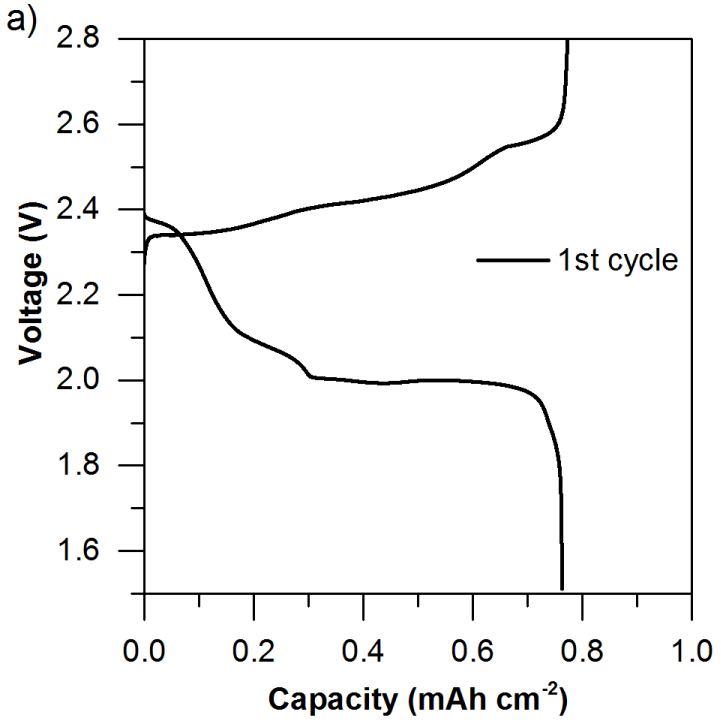

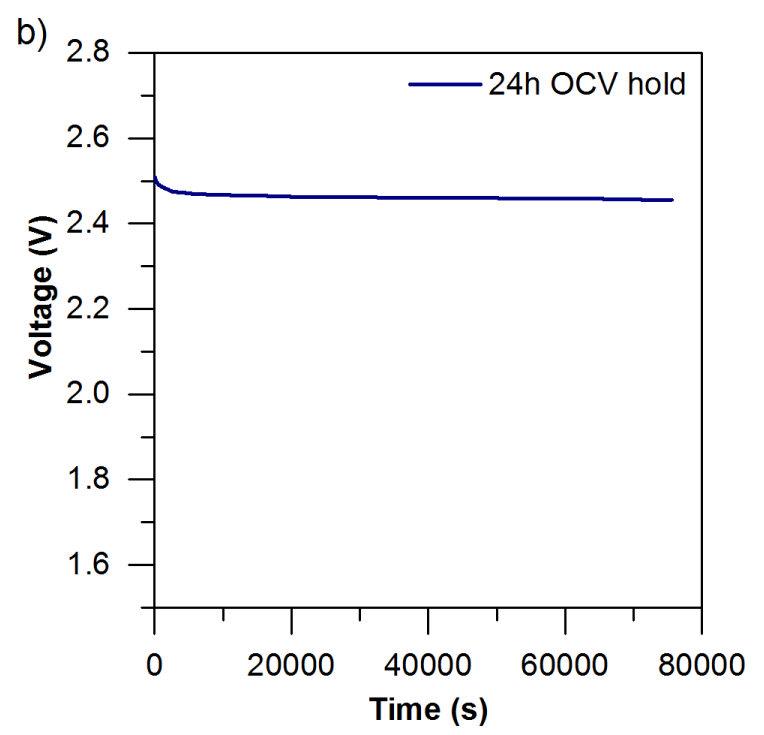

Figure 5. (a) $1^{\text {st }}$ cycle charge/discharge profile of PEEK tomography cell, in terms of areal capacities. Current rate used for tomography cell is equivalent to $\mathrm{C} / 10$ based on theoretical specific capacity of sulfur, ca. $167 \mathrm{~mA} \mathrm{~g}^{-1} \mathrm{~S}$ or $0.25 \mathrm{~mA} \mathrm{~cm}$. (b) Voltage decay of PEEK tomography cell over a 24 hour period at OCV hold. 
Measured over a continuous 24 hour period after cell assembly, the tomography cell generally exhibits stable open circuit voltage (OCV) as shown in Figure 5(b), suggesting good sealing of the cell contents from the atmosphere. The initial discharge capacity of the tomography cell, at $0.76 \mathrm{mAh} \mathrm{cm}^{-2}$, and overall profile of the discharge curve does not deviate significantly from the electrochemical cell $\left(0.97 \mathrm{mAh} \mathrm{cm}^{-2}\right)$, suggesting that the second generation PEEK cell is superior in terms of electrochemical performance compared to the original tomography cell design. It is interesting to note that after initial discharge, the tomography cell required $0.77 \mathrm{mAh} \mathrm{cm}^{-2}$ to recharge, although sulfur does not fully re-crystalize as shown in Figure 6(c), this will be discussed in a later section.

\section{$\underline{\text { The Li-S cell at various states of charge }}$}

The 3D volume rendering presented in Figure 6(a) reveals that the sulfur composite layer is no thicker than ca. $25 \mu \mathrm{m}$, introducing some challenges to segmentation and image analysis as the sulfur particles are only one layer thick. 2D reconstructed virtual slices of the elemental sulfur electrode, each at the same spatial position within the thickness of the electrode are presented in Figure 6(b,c,d), and a qualitative discussion will be made with reference to these images.

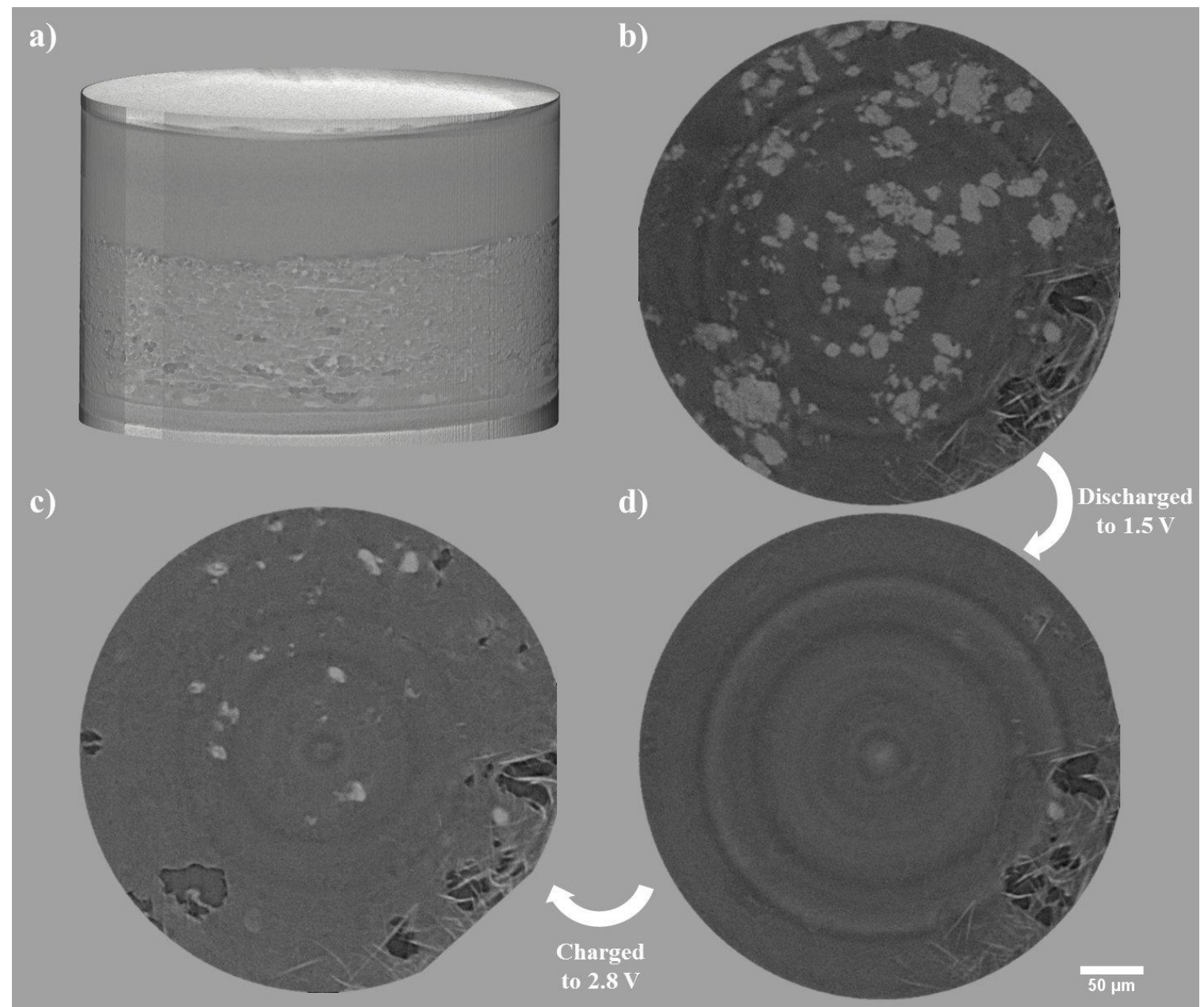

Figure 6. (a) Volume rendering of in-situ tomography cell at uncycled state. (b) 2D reconstructed virtual slice of uncycled tomography cell. (c) $2 \mathrm{D}$ reconstructed virtual slice at the same spatial position of same cell recharged to $2.8 \mathrm{~V}$. (d) $2 \mathrm{D}$ reconstructed virtual slice at the same spatial position of same cell discharged to $1.5 \mathrm{~V}$. 
Figure 6(d) reveals the almost complete disappearance of sulfur particles upon discharge to $1.5 \mathrm{~V}$. Despite this, theoretical capacity was not attained and when considered with the shortened second discharge plateau shown in Figure 5, suggests that $\mathrm{Li}_{2} \mathrm{~S}$ formation may be the capacity-limiting step. This is in addition to the polysulfide shuttle effect which resulted in the cell requiring $0.77 \mathrm{mAh} \mathrm{cm}^{-2}$ to recharge, more than $0.76 \mathrm{mAh} \mathrm{cm}^{-2}$ produced during discharge.

Whilst Figure 6(d) seems to show that only a small fraction of sulfur re-crystalizes upon charging, a search of the tomography datasets reveal that some sulfur is redeposited further away from the current collector (closer to the separator) as evidenced by Figure 7(b). In addition, the formation of nanoscopic sulfur below the spatial resolution of the image data may reduce the proportion of visible sulfur upon charging. Segmentation of the sulfur particles is complicated by the separator fibers enmeshed within the sulfur electrode as can clearly be observed in Figure 7.

Clearly, a suitable compromise for in-situ micro-CT has to be a sulfur electrode thinner than the ca. $250 \mu \mathrm{m}$ cathode used in our earlier publication (8), but thicker than the ca. $25 \mu \mathrm{m}$ cathode used in this work. In future work, elemental sulfur cathodes will be fabricated with ca. 50-100 $\mu \mathrm{m}$ dry thickness for proper image segmentation and analysis. Alternative separator materials will also be considered, such as poly-propylene films (i.e. Celgard) to simplify the segmentation process to enable further image analysis.

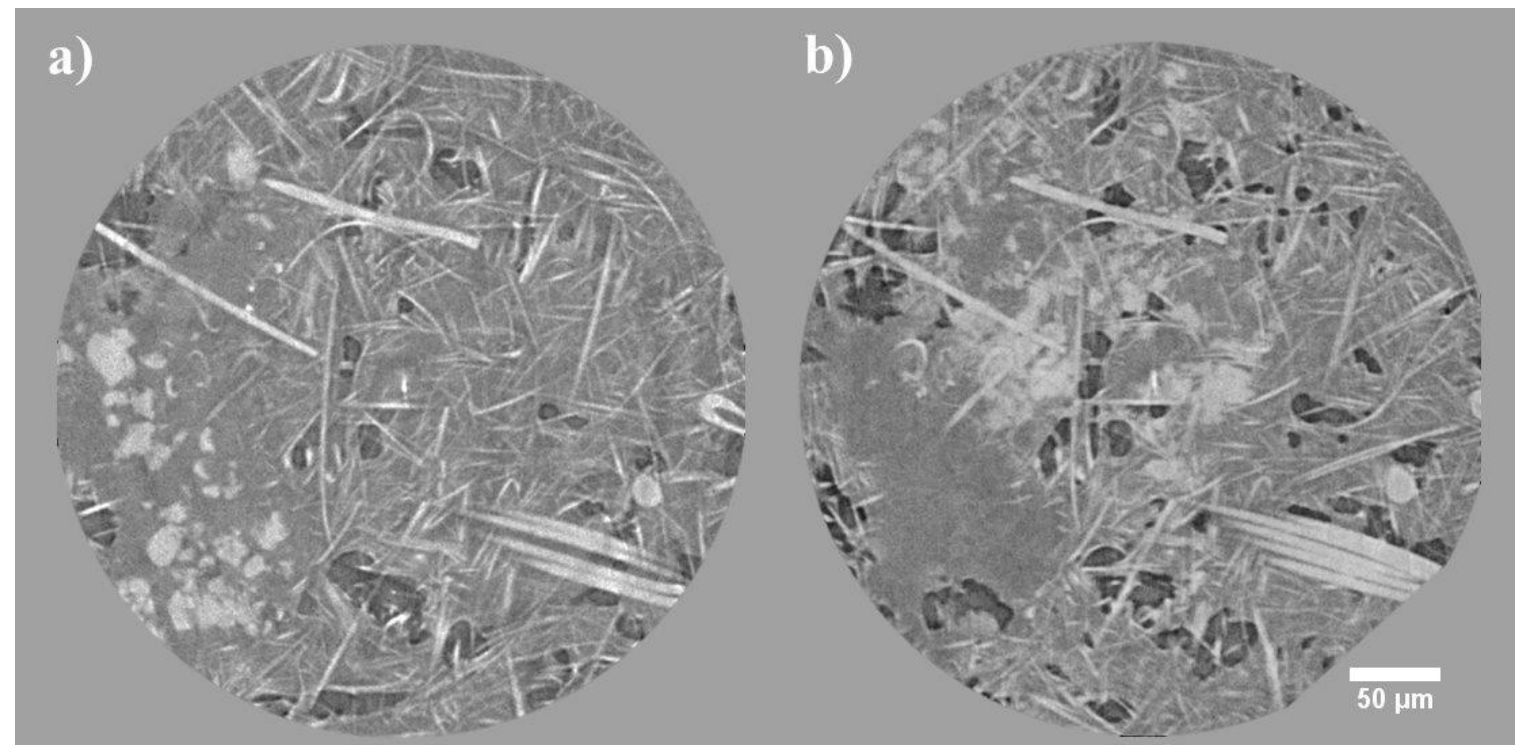

Figure 7. (a) and (b) Ortho slices at the same spatial positions (further from the current collector compared to Figure 6) of the elemental sulfur electrode at uncycled and charged states respectively.

The improvement of signal to noise ratio will also ensure that techniques such as digital volume correlation (DVC) can be more easily and more reliably applied to the tomography results. These series of advanced image analysis techniques will be explored in future work, and these preliminary results are a proof of concept on the improvements that an optimized sulfur electrode and smaller tomography cell design will bring. 


\section{Conclusions}

To conclude, a thin film elemental sulfur electrode has been imaged both $e x$-situ and in an optimized in-situ tomography cell. In the ex-situ scan, the clear phase boundaries between the carbon binder domain and pore phase within the length scale of the ex-situ micro-CT dataset raises the possibility of characterizing Li-S battery electrode properties, including porosity, tortuosity and effective electrical conductivity in future work. Finally, initial proof of concept has been established on a miniaturized PEEK tomography cell which will be developed in the near term in order to improve on image quality and shorten acquisition times.

\section{Acknowledgments}

The authors acknowledge financial support from the EPSRC (EP/K005030/1, EP/N032888/1, EP/M009394/1) and UCL and Carl Zeiss XRM.

\section{References}

1. M. Barghamadi, A. Kapoor and C. Wen, Journal of the Electrochemical Society, 160, A1256 (2013).

2. P. G. Bruce, S. A. Freunberger, L. J. Hardwick and J. M. Tarascon, Nat Mater, 11, 19 (2012).

3. Y.-c. K. Chen-Wiegart, P. Shearing, Q. Yuan, A. Tkachuk and J. Wang, Electrochemistry Communications, 21, 58 (2012).

4. Y.-c. K. Chen-Wiegart, Z. Liu, K. T. Faber, S. A. Barnett and J. Wang, Electrochemistry Communications, 28, 127 (2013).

5. M. Ebner, F. Geldmacher, F. Marone, M. Stampanoni and V. Wood, Advanced Energy Materials, 3, 845 (2013).

6. M. Ebner, F. Marone, M. Stampanoni and V. Wood, Science, 342, 716 (2013).

7. J. Wang, Y. C. Chen-Wiegart and J. Wang, Angew Chem Int Ed Engl, 53, 4460 (2014).

8. A. Yermukhambetova, C. Tan, S. R. Daemi, Z. Bakenov, J. A. Darr, D. J. Brett and P. R. Shearing, Sci Rep, 6, 35291 (2016).

9. $\quad$ R. Xu, J. Lu and K. Amine, Advanced Energy Materials, 5, 1500408 (2015). 